\title{
Hypermetabolic Lymphadenopathy on PET CT in Systemic Lupus Erythematosus: A Case Report
}

\author{
Dana DiRenzo ${ }^{1}$ and Homa Timlin ${ }^{1 *}$ \\ ${ }^{1}$ Johns Hopkins Division of Rheumatology, Baltimore.
}

Received: December 12, 2016; Accepted: January 3, 2017; Published: January 23, 2017

*Corresponding author: Homa Timlin MD MSc, Johns Hopkins Division of Rheumatology, 5501 Hopkins Bayview, Circle/Asthma and Allergy Building, Suite 1B.1, Baltimore, MD 21224,

\begin{abstract}
Background: New onset and active Systemic Lupus Erythematosus (SLE) may be infrequently associated with diffuse lymphadenopathy. This is often worrisome for malignancy in which F18/FDG PET scanning is typically obtained as part of a neoplastic workup. Results are often difficult to interpret and resemble lymphoma in many instances. There is sparse literature describing systematic differences in inflammation versus neoplasm pertaining to SLE in this context.
\end{abstract}

Case Series: We have reviewed F18/FDG PET/CT imaging and histology from three patients with hyper metabolic lymph nodes who were found to have benign pathology in the setting of active SLE.

Conclusion: FDG PET scanning is an imaging modality that is infrequently used as a means to non-invasively assess inflammation in rheumatic diseases. Further studies are needed to determine metrics for delineating benign inflammation versus neoplasm based on standard uptake units. However, lymph node biopsy should remain the gold standard for diagnosis in this particular patient population.

Keywords: PET scan; Systemic Lupus Erythematosus; Lymphadenopathy;

\section{Introduction}

Systemic Lupus Erythematosus (SLE) is an autoimmune disease that affects multiple organ systems with varied disease presentations, sometimes dramatic in onset [1]. It may be associated with lymphadenopathy, especially in instances of high disease activity [2]. Differentiating malignancies such as lymphoma versus SLE with lymphadenopathy can be difficult [3]. Of concern, there is also an increased association of non-Hodgkin's lymphoma in patients with SLE which may be associated with immunosuppressive medications versus high disease activity [4], [5]. In a recent meta-analysis of cancer risk in patients with SLE, there was a pooled Relative Risk (RR) of 5.40 (95\% CI, 3.75-7.77) for development of non-Hodgkin's lymphoma [6].

PET scanning is useful in locating areas of distant metastasis as part of cancer staging and as a translation of that, may also useful in localizing areas of inflammation [7]. The ability of PET scan to qualitatively characterize the degree of systemic inflammation and organ involvement have been previously described for SLE [8]. Mechanistically, activated lymphocytes exhibit higher degrees of glucose metabolism, concentrating in lymphoid tissues, as seen on PET scan via a radioactively labeled tracer [9].

We have reviewed three cases of new onset SLE with highly metabolic lymphadenopathy on PET scanning that had benign findings on biopsy. The PET scans were obtained out of concern for malignancy in the context of diffuse lymphadenopathy. All three PET scans posed significant angst to both the physicians and patients, despite negative biopsy results. To date, there are very limited studies assessing use of PET scan for characterizing inflammation and disease activity in patients with SLE and results may pose diagnostic dilemmas. We argue that lymph node biopsy in patients with bulky lymphadenopathy should remain the gold standard for diagnosis.

\section{Case 1}

A 33-year-old female developed progressively worsening fevers, tachycardia, pleuritic chest pain, and weight loss over several weeks' time. Imaging was repeatedly negative for pulmonary embolism but diffuse lymphadenopathy was noted on her chest CT scans. This correlated with strikingly enlarged axillary and cervical lymph nodes on her physical exam, worrisome for malignancy. She additionally developed arthralgias and was referred to our rheumatology clinic where she was found to be leukopenic with positive anti-nuclear antibodies (>1:640, homogeneous pattern), anti-dsDNA antibodies (1:320), anti-Ro antibodies, anti-La antibodies, anti-RNP antibodies, anti-Smith antibodies, low C3 of $60 \mathrm{mg} / \mathrm{dL}$ (normal 79-152 mg/dL), and low $\mathrm{C} 4 \mathrm{of}<2 \mathrm{mg} / \mathrm{dL}$. Her urine protein/creatinine ratio was elevated to 0.28 (normal $0.00-0.19$ ).

She underwent F18/FDG PET/CT scanning which demonstrated hyper metabolic cervical, axillary, mediastinal, retroperitoneal, iliac and inguinal lymph nodes with splenic and 
bone marrow involvement also concerning for lymphoma (Figure 1). Her SUVmax was 4.6. However, a fine needle aspiration of a right axillary lymph node revealed only reactive lymphoid tissue. Because the concern for lymphoma was so high, she underwent a complete excisional right axillary lymph node biopsy which was consistent with benign dermatopathic lymphadenopathy.

\section{Case 2}

A 38-year-old female with known history of systemic lupus erythematosus and overlap pauci-immune glomerulonephritis was admitted to a large tertiary care center in the setting of pancytopenia, diffuse lymphadenopathy, and 20-pound weight loss over several weeks. Her lupus was diagnosed 12 years previous to this presentation and was characterized by malar rash, photosensitivity, arthritis, pancytopenia, hypocomplementemia (nadir C3 $52 \mathrm{mg} / \mathrm{dL}$, normal 79-152 mg/ $\mathrm{dL}$; $\mathrm{C} 4 \mathrm{mg} / \mathrm{dL}$, normal $12-42 \mathrm{mg} / \mathrm{dL}$ ), anti-nuclear antibodies (1:160), anti-dsDNA antibodies (1:640), and anti-RNP antibodies. She was also noted to be short of breath with progressively worsening cough. She had CT imaging of her chest, abdomen, and pelvis which revealed periportal, peripancreatic, retroperitoneal, axillary, and supraclavicular lymphadenopathy. A F18/FDG PET/CT scan was obtained and revealed diffuse, highly metabolic lymphadenopathy. One hyperintense subcarinal lymph node measured 13.2 SUVmax. However, a supraclavicular excisional lymph node biopsy was performed which showed histiocytic lymphoproliferation with coagulative necrosis consistent with lupus lymphadenitis.

\section{Case 3}

A 25-year-old female developed axillary and cervical lymphadenopathy, recurrent pleurisy, and severe arthritis over several months' time. She was referred to our rheumatology clinic where her immunologic studies were notable for anti-nuclear
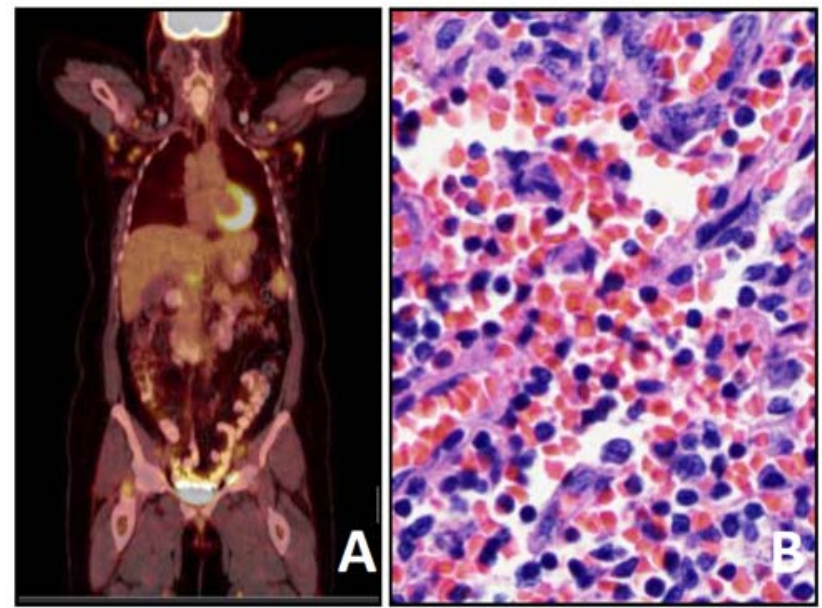

Figure 1: A) F18/FDG PET Scan from Case 1 demonstrating diffuse hyper metabolic cervical, axillary, mediastinal, retroperitoneal, iliac, and inguinal lymph nodes with splenic and bone marrow involvement.

B) Histology of right axillary lymph node demonstrating reactive lymphoid tissue without evidence of malignancy. antibodies ( $>1: 640)$, anti-Smith antibodies, anti-dsDNA (1:20), and anti-SSA and anti-SSB antibodies. She had low complements with a C3 of $58 \mathrm{mg} / \mathrm{dL}$ (normal 79-152 mg/dL), and low C4 of 3 $\mathrm{mg} / \mathrm{dL}$ (normal $12-42 \mathrm{mg} / \mathrm{dL}$ ). She was subsequently diagnosed with SLE. Because of persistent axillary lymphadenopathy over serial examinations, there was concern for a neoplastic process and further imaging was obtained. A F18/FDG PET CT showed hyper metabolic activity isolated to her axillary lymph nodes. The reported SUVmax was 4.1. She underwent a fine needle aspiration of a right axillary node which revealed reactive lymphoid tissue with follicular and paracortical hyperplasia.

\section{Discussion}

Each of these cases represents development of bulky lymphadenopathy in the context of SLE that generated concern for lymphoma. All three of these cases displayed hyper metabolic lymph nodes on PET scanning, but without congruent features or intensities. While PET scanning may theoretically be used as a non-invasive modality for diagnosing inflammation versus malignancy, it lacks the precision for defining these very different entities. We argue that biopsy should remain the gold standard for assessing lymphadenopathy in lupus patients, a population at higher risk for lymphoma.

PET scanning may be used to differentiate malignancy versus inflammation in other entities, but current literature is limited to case reports in terms of SLE. In a retrospective analysis of 32 patients with Sjogren's Syndrome, 4 patients developed lymphoma and their imaging was notable for relatively higher standard uptake unit (SUV) max compared to those with solely reactive lymphadenopathy (5.4 vs 3.2 SUVmax, $p=0.05$ ). A study analyzing 48 patients with Fever of Unknown Origin (FUO) noted the mean SUV reached 10.4 (range 7.2-15.3) in tumors, 3.8 (range 3.2-5.6) in inflammation respectively [10]. However, we noted that in Case 2, biopsied to be benign lupus lymphadenitis, the SUVmax was 13.2. Importantly, PET scanner and reconstruction methods are factors that intimately affect SUV measurements and stringent quality control measures are necessary when comparing studies [11].

Another high risk feature noted on PET scan concerning for lymphoma is bone marrow infiltration. In a meta-analysis of patients with newly diagnosed diffuse large B-cell lymphoma, positive FDG PET/CT findings of bone marrow involvement negated the need for a bone marrow biopsy for the detection of bone marrow involvement in these patients [12].

\section{Conclusion}

There is an additional need for larger scale, high powered studies to better define measurements to easily delineate inflammation versus malignancy on PET scanning. While this would idealistically serve to avoid invasive surgical procedures, the gold standard for diagnosis in these high risk patients should remain lymph node biopsy. These cases serve to demonstrate the high burden of inflammation in developing SLE that is variable in 
nature.

\section{References}

1. Soediono B. Systemic Lupus Erythematosus. J Chem Inf Model. $1989 ; 53: 160$

2. Shapira Y, Weinberger A, and Wysenbeek AJ. Lymphadenopathy in systemic lupus erythematosus. Prevalence and relation to disease manifestations. Clin Rheumatol.1996;15(4):335-338.

3. Melikoglu MA and Melikoglu M. LAP as a Manifestation of SLE. Acta Reum Port. 2008;33:402-406.

4. Bernatsky S, Ramsey-Goldman R, Joseph L, Boivin JF, Costenbader, Urowitz MB, et al. Lymphoma risk in systemic lupus: effects of disease activity versus treatment." Ann Rheum Dis. 2014;73(1):138-142.

5. Bichile $\mathrm{T}$ and Petri $\mathrm{T}$. Incidence of lymphoma associated with underlying lupus: lessons learned from observational studies. Curr Opin Rheumatol. 2014;26:111-117, 2014.

6. Cao L, Tong $\mathrm{H}, \mathrm{Xu} \mathrm{H}$, Liu H, Meng H, Wang J, et al. Systemic lupus erythematous and malignancy risk: A meta-analysis. PLoS One. 2015;10(4):1-21. doi: 10.1371/journal.pone.0122964.

7. Yamashita H, Kubota K. Mimori A. Clinical value of whole-body PET/ CT in patients with active rheumatic diseases. Arthritis Res Ther
$2014 ; 16(5): 423$.

8. Curiel R, Akin EA, Beaulieu G, Depalma L, Hashefi M. PET/CT imaging in systemic lupus erythematosus. Ann N Y Acad Sci. 2011;1228(1):7180. doi: 10.1111/j.1749-6632.2011.06076.x.

9. Nowak M, Carrasquillo a J, Yarboro CH, Bacharach SL, Whatley, Valencia X, et al. A pilot study of the use of 2-[18F]-fluoro-2-deoxyD-glucose-positron emission tomography to assess the distribution of activated lymphocytes in patients with systemic lupus erythematosus. Arthritis Rheum. 2004;50(4):1233-1238.

10. Ferda J, Ferdová E, Záhlavav J, Matějovič M, Kreuzberg B. Fever of unknown origin: A value of 18F-FDG-PET/CT with integrated full diagnostic isotropic CT imaging. Eur J Radiol. 2010;73(3):518-525. doi: 10.1016/j.ejrad.2008.12.014.

11. Adams MC, Turkington TG, Wilson JM, and Wong TG. A systematic review of the factors affecting accuracy of SUV measurements. Am J Roentgenol. 2010;195(2):310-320. doi: 10.2214/AJR.10.4923.

12. Adams HJA, TC Kwee, De Keizer B, Fijnheer R, De Klerk JMH, Nievelstein RAJ. FDG PET/CT for the detection of bone marrow involvement in diffuse large B-cell lymphoma: Systematic review and meta-analysis. 